\title{
El Sistema Alimentario Mexicano: su acción en el campo y en la alimentación, 1980-1982
}

\author{
Luis Ozmar Pedroza Ortega'
}

Recibido: 13 de abril de 2018 - Aprobado: 20 de julio de 2018

\begin{abstract}
Resumen
El Sistema Alimentario Mexicano (SAM) fue una política pública destinada a atender las necesidades alimentarias de la población, así como la incentivación a la producción y consumo de alimentos básicos en México durante los primeros años de la década de 1980. El objetivo de esta investigación es analizar la propuesta en materia de alimentación y producción del SAM e indagar las repercusiones de su puesta en marcha para hacer frente a la crisis económica y agrícola que había azotado al campo mexicano desde finales de la década de 1970. El artículo se fundamenta en prensa de la época y con documentos operativos del programa. Este estudio contribuye a la historiografía de la alimentación al abordar los cambios en la producción alimentaria de México durante la segunda mitad del siglo xx.
\end{abstract}

Palabras clave: alimentación, crisis agrícola, campo mexicano, nutrición.

\section{The Mexican Food System:}

Its action in the countryside and in alimentation, 1980-1982

\begin{abstract}
The Mexican Food System (SAM) was a public policy designed to meet the population's food needs, as well as to encour age production and consumption of basic foods in Mexico during the first years of 1980s. This research is aimed at analyzing SAM's proposal on food and production and repercussions of its implementation facing the economic and agricultural crisis for the Mexican countryside since the end of 1970s. The article is based on press news of that time and programmatic operative documents. This study contributes to the historiography of food by addressing changes in food production in Mexico during the second half of the 20th Century.
\end{abstract}

Keywords: food, agricultural crisis, Mexican countryside, nutrition.

1 Mexicano. Doctorando en Historia Moderna y Contemporánea, Instituto de Investigaciones Dr. José María Luis Mora, México. E-mail: ozmarpedroza@gmail.com 


\title{
0 Sistema Alimentar Mexicano: sua ação no campo e na alimentação, 1980-1982
}

\begin{abstract}
Resumo
O Sistema Alimentar Mexicano (SAM) foi uma política pública destinada a atender as necessidades alimentares da população, assim como o incentivo à produção e consumo de alimentos básicos no México durante os primeiros anos da década de 1980. 0 objetivo desta pesquisa é analisar qual foi a proposta em matéria de alimentação e produção do SAM e indagar quais foram as repercussões que teve sua implementação para lidar com a crise econômica e agrícola que tinha afetado ao campo mexicano desde finais da década de 1970. 0 artigo fundamenta-se na imprensa da época e com documentos operativos do programa. Este estudo contribui à historiografia da alimentação a abordar as mudanças na produção alimentar do México durante a segunda metade do século xx.
\end{abstract}

Palavras-chave: alimentação, crise agrícola, campo mexicano, nutrição.

Durante gran parte de la década de 1970, México afrontó una crisis agrícola que, al igual que en muchos países de América Latina, se propagó a otras esferas como la de la producción de alimentos básicos. Después de una década de acelerado crecimiento y desarrollo económico, el sector agrícola empezó a dar signos de bajo rendimiento en la producción. Para 1973 el país estaba perdiendo la autosuficiencia en maíz y se corría el riesgo que también fuera dependiente en otros productos, como el trigo y el frijol.

En esta disyuntiva, el gobierno creó en 1980 el Sistema Alimentario Mexicano (SAM). Este programa aparecía como innovador y esperanzador, pues aseguraba que el Estado rechazaría medidas de libre comercio y en su lugar apostaría a reavivar el sector de alimentos básicos y a reducir la dependencia en las importaciones de este tipo de productos. La puesta en marcha de este programa suponía la reorientación de las prioridades del desarrollo del sector agrícola; por ello, esta política ocasionó grandes debates. Además, puso de manifiesto otros problemas en el campo mexicano, como los bajos niveles de vida de la población campesina, la concentración de la tierra y la lucha por el acceso a ésta.

En este contexto, el SAM se presentó como una estrategia gubernamental destinada principalmente a incrementar la producción agrícola, lo cual lograría subsanar otro tipo de problemáticas, como el rezago técnico y la subalimentación en el campo. Fue diseñado por la Oficina de Asesores de la presidencia de la república -bajo el mandato de José López Portillo-y, por esta razón, su marco de acción y objetivos exigieron la participación de múltiples instancias gubernamentales y organizaciones agrícolas - privadas y ejidales- para lograr dos metas esenciales a mediano plazo: la autosuficiencia alimentaria del país y el combate a la situación de pobreza en la que se hallaba la mayoría de la población rural (López, 1980: 4-5). 
Cabe destacar que la creación del SAM estuvo condicionada por el ambiente de auge económico propiciado por el descubrimiento de yacimientos petroleros a finales de la década de 1970. Esta ilusión financiera permitió que parte de los excedentes generados por el petróleo se convirtieran en recursos transferidos al sector agrícola, con el fin de impulsar su modernización y el desarrollo nutricional de las zonas rurales (López, 1980: 6). Debido a su gran peso en el presupuesto del gobierno federal, el SAM fue uno de los primeros programas en ser cancelados en 1982, momento en el que estalla una nueva crisis económica.

La pregunta que guía y busca responder este trabajo es ¿cuáles fueron las circunstancias político-económicas en las que se desarrolló el Sistema Alimentario Mexicano (SAM) y cuál fue su papel como política alimentaria? En este sentido, se afirma que el SAM surgió como un proyecto que pretendía constituirse como una salida a la crisis agrícola que atravesó el país desde la segunda mitad de la década de 1970. Esta crisis se manifestó a través de la pérdida de la autosuficiencia en maíz y la marginación de gran parte de la población rural. El SAM proponía combatir estos dos grandes problemas con base en un programa de subsidios a la producción y al consumo, la equidad en el abasto y en la distribución de alimentos básicos.

El objetivo del trabajo es analizar el diseño y los efectos programáticos más importantes del SAM, como un proyecto gubernamental orientado a superar la crisis alimentaria de finales de la década de 1970. El análisis se realiza con base en tres apartados. En primer lugar, el estudio de la crisis agrícola, como antecedente inmediato y contexto en el que surge el SAM. En segundo lugar, el análisis de las propuestas específicas de este proyecto en el plano operativo, para ello se utilizan algunos documentos oficiales sobre el diseño del programa, fuentes hemerográficas que siguieron el desempeño y las acciones del proyecto en la prensa y se complementa con estudios que se han realizado sobre el $\mathrm{SAM}^{2}{ }^{2}$ los cuales resultan escasos, además de que han sido en su mayoría economistas y sociólogos los que se han adentrado tema, así que existe un vacío historiográfico que es necesario empezar a trabajar. Por último, se identifican las posibles causas que hicieron fracasar el SAM, así como se indican qué efectos en la producción y abasto de alimentos básicos produjo. Cabe destacar que también se resaltan los elementos políticos y económicos que puedan explicar qué circunstancias llevaron al abrupto fracaso que tuvo el programa en 1982.

2 Los principales autores que se han dedicado al tema del Sistema Alimentario Mexicano han sido Rose Spalding, quien se ha dedicado a analizar esta política desde una visión sociológica, y Cassio Luiselli -que carga con el sesgo de haber sido su director general-, que ha descrito el funcionamiento y las aspiraciones que tuvo el programa (Spalding, 1985; Luiselli, 2017). 
Por último, la importancia de estudiar el SAM reside en aportar al debate que generó este programa como política pública destinada a mejorar la alimentación de la población mexicana de escasos recursos entre 1980 y 1982. Asimismo, busca contribuir a la historiografía de la alimentación, pues el SAM se ha analizado desde la economía y sociología, mientras que ha estado ausente en la historiografía. Al abordar el tema como un proceso histórico, es posible arrojar luz sobre la importancia de las relaciones de este proyecto con otros problemas sociales, como la tenencia de la tierra, la nutrición, la economía y la política, argumentos que reafirman la condición interdisciplinar del estudio de la alimentación durante el siglo xx.

\section{La crisis agrícola en México en la segunda mitad del siglo $\mathrm{xx}$}

El siglo xx mexicano es un siglo corto en cuestión agrícola. Arranca en 1920 con el fin del conflicto revolucionario y la emergencia de un nuevo Estado con un profundo cariz agrario, y culmina con la reforma agraria de 1992 y la movilización indígena zapatista, basada en una reinterpretación de las exigencias campesinas y agrarias provenientes de la revolución (Luiselli, 2017: 103). En el mundo, el siglo $x x$ fue un periodo de grandes avances agrícolas. La agricultura se vio fortalecida por la aplicación de nuevas prácticas de cultivo, el diseño de nuevos sistemas de irrigación y el desarrollo de la industria agroquímica. Asimismo, se desarrolló la Revolución Verde que, además de los agroquímicos y el riego, introdujo variedades de semillas mejoradas que incrementaron los rendimientos de los principales cereales. México no fue ajeno a estos cambios, sino que se convirtió en la misma cuna de la Revolución Verde, al contar con el Centro Internacional de Mejoramiento del Maíz y Trigo (CIMMYT). No obstante, estos avances tecnológicos tuvieron un impacto limitado y desigual en el campo mexicano. Para comprender la situación de crisis agrícola y económica en la que estaba el país para la década de 1970, es necesario exponer algunos puntos importantes sobre el desarrollo agrícola mexicano y los vaivenes en la política agraria.

\section{El desarrollo agrícola mexicano: un breve preámbulo a la crisis}

Desde finales de la década de 1920, con una nueva élite gobernante auspiciada bajo la égida del partido del régimen, se dieron grandes cambios estructurales que dotaron al nuevo Estado de los instrumentos necesarios para sentar las bases para el crecimiento económico y el control social en el país. Además de la política, la economía se convirtió en un asunto urgente para los gobiernos posrevolucionarios, pues dependía de ella mantener la 
pacificación lograda luego del conflicto revolucionario, a través de impulsar mejoras en las condiciones de vida de la población.

El sexenio de Lázaro Cárdenas (1934-1940) marcó un viraje del régimen mexicano. El Partido Nacional Revolucionario (PNR), la primera agrupación unipartidista institucional, se transformó en el Partido de la Revolución Mexicana (PRM). Esta institución implantó una política de masas en la que aglutinó a los sectores sociales bajo un enfoque corporativista, incentivando la participación política, siempre y cuando estuviera alineada al gobierno. Con ello buscó mantener el consenso político y centrarse en procesos económicos y sociales para impulsar el crecimiento y desarrollo del país.

Así, en la cuestión agraria, se alentó la organización campesina, al crear la Confederación Nacional Campesina (CNC) con el objetivo de institucionalizar el reparto de tierras y la unificación de las exigencias agrarias (El Universal, 11-VII-1935). Es importante resaltar que esta agrupación sirvió como un eficaz mecanismo de control político del campesinado por parte del gobierno, pues buscó regularizar la tenencia de la tierra y contener las demandas de los campesinos. En estos años, también se fundaron las primeras instituciones dedicadas a atender los problemas del campo. Con ellas se dieron los primeros apoyos gubernamentales a los productores agrícolas. Dicha ayuda se dio por medio del Banco Nacional de Crédito Ejidal y la Comisión Nacional Agraria, instancias que buscaron incentivar la producción para la exportación; mientras el abasto para el consumo interno de alimentos básicos dependió de la creación de los Almacenes Nacionales de Depósito S. A. (ANDSA) y los comités de regulación de granos.

Las décadas de 1940 y 1950, en la que sucedieron los gobiernos de Manuel Ávila Camacho (1940-1946), Miguel Alemán (1946-1952) y Adolfo Ruiz Cortines (1952-1958), no solo fue la época en la que se dio una nueva reforma política al fundarse, con base en el PRM, el Partido Revolucionario Institucional (PRI), entidad que detentaría el poder durante los años que siguieron, y que fue la institución desde la que se ejerció un férreo control político de los sectores sociales, como obreros y campesinos. Asimismo, esta época marcó el mayor crecimiento agrícola del país. Hubo varios factores que detonaron esta situación, En 1940 el país contaba con poco más de 20 millones de habitantes y era sumamente rural; para 1960 había 34 millones y el medio urbano empezaba a ensancharse (Luiselli, 2017: 116), tal situación hizo necesario aumentar la producción agrícola interna para contar con alimentos básicos para la población que iba en aumento. De esta forma, el mercado interno empezó a crecer en demanda, por lo cual hubo una expansión de la frontera agrícola, es decir, se ampliaron las tierras de cultivo que se combinaron con las grandes obras de irrigación que se emprendieron por parte del gobierno. Además, se adoptó el modelo económico de sustitución 
de importaciones con el que se dio un impulso a la industrialización. Dicho modelo apoyó a los campesinos interesados en producir para satisfacer la demanda del creciente mercado interno, y disminuyó su ayuda a los agroexportadores. No obstante, este reacomodo de intereses permitió que surgieran nuevos empresarios agrícolas con una gran capacidad productiva y con recursos financieros disponibles para sostener nuevas zonas de producción. Este fue el inicio de la modernización de la agricultura en México y significó la reconfiguración productiva del país. Las zonas tradicionales de cultivo en el centro y sur dieron paso a nuevos centros de producción ubicados en el norte, que se beneficiaron por las obras de riego y por la transferencia de recursos crediticios y tecnológicos.

\section{Revolución Verde en México: modernización e investigación agrícola}

Tal como menciona Cynthia Hewitt (1988), la investigación agrícola en México dio comienzo, en la década de 1930, con la fundación del Departamento de Estaciones experimentales de la Secretaría de Agricultura, el cual se dedicó a recoger muestras de semillas de trigo y maíz para encontrar variedades de mayor rendimiento. La convicción de este grupo comprendía que solo se alcanzaría una mayor productividad en el campo mexicano a través de cambios estructurales que transformarían las propiedades capitalistas en cooperativas operadas por campesinos y jornaleros (Hewitt, 1988: 31).

Para 1947, este pequeño departamento se consolidó y convirtió en el Instituto de Investigaciones Agrícolas (IIA), que operó hasta 1960. A la par de esta nueva institución, se incorporó a la Oficina de Estudios Especiales (OEE) el programa conjunto de asistencia e investigación agrícola del gobierno mexicano y la Fundación Rockefeller. Dicho programa tenía el objetivo de realizar pruebas para incrementar la producción en el sector privado de la agricultura, dejando el problema de las pequeñas parcelas y la provisión de alimentos básicos a las actividades realizadas por el IIA. Ambas instituciones tuvieron ideas distintas sobre los objetivos finales de sus investigaciones agrícolas. Mientras la OEE se dedicó a analizar las implicaciones socioeconómicas de la maximización de los rendimientos agrícolas, mediante la aplicación de nueva tecnología, como la adaptación de semillas a los suelos locales, la aplicación de insecticidas y fertilizantes y el uso eficiente del agua, el IIA buscaba obtener semillas mejoradas -primordialmente de maíz- para zonas de cultivos tradicionales (Hewitt, 1988: 33).

En 1961, luego de haber existido en paralelo y manejado una agenda de investigación dispar, el IIA y la OEE se fusionaron para crear el Instituto Nacional de Investigaciones Agrícolas (INIA). Las finalidades de esta entidad fueron permeadas por los trabajos de la OEE, por lo que se enfocaron en 
la agricultura comercial, relegando a un segundo plano los problemas de la agricultura de subsistencia y de temporal. Esto significó la consolidación del proyecto de transferencia de tecnología agrícola de Estados Unidos a México, con el fin de impulsar el rendimiento del agro mexicano orientado a la exportación.

Este panorama de investigación agrícola en México fue el escenario en el que se dieron una serie de experimentos biológicos, químicos y técnicos que incentivaron una nueva forma de comercio agrícola, tal coyuntura se le denominó Revolución Verde. Para Hewitt, esta revolución fue un proceso de modernización efectuado en las principales zonas agrícolas comerciales de México desde 1940. La finalidad de este proceso era el aumento de la productividad, por lo que se ha requerido del uso de semillas de alto rendimiento, fertilizantes, insecticidas, herbicidas, maquinaria agrícola e irrigación. Tales insumos solo se obtuvieron mediante la ampliación del crédito y nuevos modelos económicos de compra y venta de granos y cereales.

En este sentido, la Revolución Verde en México significó un proceso de modernización de la agricultura, auspiciado por la investigación agrícola y por la transferencia de tecnología por medio del financiamiento crediticio. Esta revolución fue una de las causas del acelerado desarrollo agrícola mexicano entre 1940 y 1965. Sin embargo, los beneficios productivos de la Revolución Verde son pocos si se compara con los costos sociales, económicos y ecológicos que provocó para el país. La modernización agrícola permitió la concentración de los recursos en un puñado de titulares, los cuales no fueron capaces de producir los alimentos necesarios para la población en expansión, mientras que la presión cayó en las zonas de agricultura tradicional que, al verse limitadas tanto en recursos financieros como tecnológicos, no pudieron hacer frente a la ineficiencia agrícola del sistema productivo mexicano.

Desde 1965 a 1976, se agudizó el problema agrícola. La demanda de alimentos e insumos rebasó a la oferta. El campo mexicano entró en una crisis productiva y la presión por dotación de tierras se hizo más tensa. La CNC se ve superada al crearse nuevas asociaciones de campesinos no vinculadas al partido oficial (el PRI) y con demandas agrarias sólidas. El gobierno respondió con un reparto de tierras, incluso más grande que el llevado a cabo por Cárdenas, aunque dicho reparto fue de terrenos de baja calidad para el cultivo. La consecuencia más clara fue que la mayoría de los campesinos, al verse rezagados por las malas cosechas y las negativas a acceder a apoyos del gobierno, se volcaron a una economía de subsistencia; esto provocó, a su vez, que la crisis agrícola y económica se expandiera a otras esferas de la cadena de producción de alimentos. Esta situación se volvió insostenible para el gobierno mexicano, el cual convino resolver el problema agrícola con una nueva política agraria que incentivaría la producción y buscaría disminuir las 
importaciones de grano. Era el momento, ante una coyuntura mundial que coincidió, de recobrar la autosuficiencia en alimentos.

\section{Los años de la crisis y la tentativa respuesta}

Cuando la FAO llevó a cabo su cuarta Encuesta Alimentaria Mundial en 1977 sobre la situación del hambre y la desnutrición en el mundo, encontró que entre el 10 y 15 por ciento de la población mundial estaba subalimentada, mientras que el 50 por ciento sufría desnutrición y hambre (O'Broin, 2015: 30). En consonancia con esta aguda realidad, esta organización instó a los gobiernos de sus países miembros a crear planes para alcanzar la autosuficiencia alimentaria, particularmente en los países en vías de desarrollo, a través de la creación en 1977 del sistema mundial de información y alerta sobre la alimentación y la agricultura (O'Broin, 2015: 30). Este instrumento sirvió para empezar a hacer frente a los problemas agrícolas que se agudizaron con la crisis de 1976. Entre las principales medidas que recomendaba se hallaban la promoción de técnicas agrícolas, como el riego a pequeña escala, la ampliación de la frontera agrícola, el uso de tierras de temporal, el aprovechamiento de los recursos marinos y el aumento de la producción ganadera intensiva (O'Broin, 2015: 33-33). Estas políticas se reforzaron en 1979 con la Conferencia Mundial sobre Reforma Agraria y Desarrollo Rural celebrada en Roma, ${ }^{3}$ en donde se adoptó la "Carta del Campesino" para garantizar que la gente en condiciones de pobreza tuviera acceso a las tierras para contar con un ingreso que les permitiera alimentarse (O'Broin, 2015: 30).

Acorde con el contexto internacional, en México también se vivía una situación de alarma debido a la contracción de la economía que se había producido con la crisis de 1976. Enrique Cárdenas asevera que dicha situación provino de la incompatibilidad entre el número de objetivos y el número de instrumentos de política económica implementados, ya que en la administración de Luis Echeverría se agregó un objetivo más a la política económica de "desarrollo compartido": la distribución del ingreso. Sin embargo, no se instrumentó una medida adicional para aplicar dicha aspiración, como la reforma tributaria necesaria, lo cual provocó un desequilibrio que llevó a

3 La FAO patrocinó diversos encuentros entre los representantes de sus países miembros para discutir las medidas que se tomarían para mitigar el revés que había sufrido la agricultura mundial desde inicios de la década de 1970. Además de las políticas señaladas, también hubo una preocupación por los embates del cambio climático que se empezaban ya a notar para esa época; de ahí que la FAO se preocupara por difundir la necesidad de que los gobiernos y los organismos internacionales tuvieran que trabajar más estrechamente en diferentes sectores para erradicar el hambre y estimular el apoyo público. Por ello se conoce a este proyecto como "Unidos en la lucha contra el hambre". 
la fuga de capitales, recesión bancaria y a la devaluación del peso el 31 de agosto de 1976 (Cárdenas, 2015: 627-628).

La depresión económica solo agudizó la situación del campo mexicano. Hubo un desplome en los rendimientos en la producción de este sector, por lo que su contribución a la balanza comercial agropecuaria y al sector de las finanzas públicas empezó a disminuir (Yúnez, 2010: 738). Aunado a esto, el aumento de la población y de la demanda de alimentos ocasionó un descenso en las exportaciones y un incremento en las importaciones. De tal manera que esta crisis agrícola se identificó con la incapacidad del sector para satisfacer las necesidades alimentarias de la población, así como para autoabastecer al país de granos básicos y oleaginosas (Lustig y Pérez, 1982: 248). Tan solo deben revisarse las cifras que muestran el aumento vertiginoso de las importaciones de maíz, trigo y frijol para corroborar la insuficiencia alimentaria por la que atravesaba el país (véase la tabla 1). Para 1976 ya se importaban más de 900 mil toneladas de trigo y un millón de toneladas de maíz, así como cantidades menores para el frijol, sorgo y soya. Cabe destacar que los elementos políticos y económicos que explican la crisis agrícola no solo respondieron a la situación mundial que trataba de controlar la FAO, sino que existieron distintos factores internos que agravaron la ya frágil circunstancia agrícola mexicana. Así, es esencial destacar que dichos elementos fueron las bajas condiciones de vida de la población campesina, la concentración de la tierra en pocas manos y el conflicto entre el Estado y el campesinado por el acceso y distribución de la misma.

Tabla 1

Importaciones de granos básicos 1976-1989

(Toneladas)

\begin{tabular}{|c|r|r|r|}
\hline Año & \multicolumn{1}{|c|}{ Maíz } & \multicolumn{1}{c|}{ Trigo } & \multicolumn{1}{c|}{ Frijol } \\
\hline 1976 & 913.786 & 5.331 & 879 \\
\hline 1977 & 1.985 .619 & 456.373 & 29.256 \\
\hline 1978 & 1.344 .404 & 458.501 & 1.220 \\
\hline 1979 & 746.278 & 1.169 .006 & 6.786 \\
\hline 1980 & 4.187 .072 & 932.469 & 443.066 \\
\hline
\end{tabular}

Fuente: Informe Anual 1981, Banco de México, S. A.; Lustig, 1982: 249.

Sobre las condiciones de vida, la Secretaría de Programación y Presupuesto (SPP) realizó una Encuesta de Ingresos y Gastos de los Hogares en 1977, la cual arrojó que de cerca del 45 por ciento de la población que percibía un ingreso, éste era inferior al salario mínimo correspondiente, además de concentrarse este hecho en el sector rural (Lustig y Pérez, 1982: 250). Por lo 
tanto, la población más pobre del país se componía de campesinos y jornaleros, quienes no contaban con tierra propia o, si lo hacían, su producción era de autoconsumo. Es importante resaltar que esta desigualdad social entre los estratos y las diferencias abismales de ingresos configuraban patrones de gasto y consumo doméstico muy diferentes. Esta situación también provocaba niveles dispares de nutrición porque la dieta de los estratos rurales se mantenía en el nivel de subsistencia.

Este hecho era consecuencia inevitable de la concentración de la tierra en algunos titulares. En el medio rural mexicano de finales de los setenta había agroindustriales que acaparaban gran parte de las tierras de cultivo, dejándoles a los pequeños campesinos los terrenos menos fértiles que no producían los alimentos suficientes para subsistir. Por ello, la situación campesina era desafortunada; había un estancamiento de ingresos, subempleo, proletarización y migración temporal y permanente (Durston, 1981: 4). Como resultado, empezó a generalizarse un descontento social entre los campesinos y trabajadores agrícolas, que no veían reflejado su trabajo en sus condiciones de vida y en la preocupación del Estado por procurarles sus derechos básicos como ciudadanos.

La mínima intervención de la población rural en el desarrollo nacional y que provocó el conflicto entre el campesinado y el Estado, puede explicarse a partir de la reflexión de las formas de participación agrícola que se dieron desde los regímenes posteriores a 1940. A partir de esta fecha puede apreciarse cómo empezó, por un lado, una serie de subsidios a la agricultura capitalista orientada a la exportación a través de inversión pública en infraestructura, investigaciones agronómicas y establecimiento de medidas proteccionistas como los precios garantía para el comercio exterior (Cárdenas, 2015: 596). ${ }^{4}$ Estos subsidios estaban destinados a impulsar la zona noroeste del país donde la agricultura comercial se desarrollaba plenamente, además de fomentar el uso de semillas mejoradas de alto rendimiento producidas por la llamada Revolución Verde; bajo el discurso de lograr la autosuficiencia alimentaria, ${ }^{5}$ se defendía el aumento de la producción (Navarro, 1988: 103). Por otro lado, los pequeños agricultores se dedicaron a desempeñar el papel de proveedores de materias primas -maíz y frijol principalmente- que comercializaban a bajo precio en los centros urbanos. A estos pequeños pro-

4 La política de precios de garantía, administrados por la Compañía Nacional de Subsistencias Populares (CONASUPO), mantuvo precios fijos por mucho tiempo, lo que provocó un reducido interés por invertir en el campo.

5 Es necesario aclarar que el término "autosuficiencia alimentaria" era ocupado por el gobierno mexicano como sinónimo de "seguridad alimentaria". Ambos conceptos se definían como la posibilidad de acceso continuo en el tiempo y en el espacio a los alimentos esenciales para el desarrollo y funcionamiento normal de los individuos en sociedad. 
ductores también se les negó el acceso a los medios crediticios, financieros y técnicos para que impulsaran su producción, sin contar que fueron los que más resintieron las medidas antiinflacionarias establecidas para contrarrestar la crisis económica de 1976, lo que llevó al gobierno a mantener bajos los precios de las cosechas para no disparar los costos de alimentos básicos en las ciudades (Durston, 1981: 4).

Con este panorama, es posible afirmar que la crisis económica que produjo a su vez la emergencia agrícola y alimentaria a finales de la década de 1970 provino de las medidas estatales en torno al fomento de un tipo de agricultura -la comercial y con orientación exportadora- en detrimento de otras formas de producción, como los distritos de temporal, aquellas unidades productivas de la gran mayoría de los campesinos mexicanos durante esa época. Por esta razón, es plausible aseverar que la subordinación de la agricultura al desarrollo industrial y a las tendencias internacionales, implicaron la transferencia de excedentes del sector agrícola tradicional en años anteriores, a la agricultura capitalista y a otras actividades productivas (Lustig y Pérez, 1982: 265). Estas medidas de política económica expandieron formas de producción agroindustrial y mermaron el trabajo manual de los campesinos ejidatarios. Cuando se presentaron las consecuencias de la contracción de la economía, el deterioro en la estructura productiva del campo volvió más apremiante crear una política que se dedicara a reajustar y reactivar la producción de los alimentos básicos, pues la crisis y las mismas políticas habían provocado que el país no fuera capaz de alimentar a su propia población.

La idea que fomentó el Estado estuvo vinculada con la emergencia internacional sobre el problema del hambre que la FAO había promovido y la importancia que tenía la acción directa de los gobiernos para resolver los déficits nutritivos y de producción de alimentos básicos (O'Broin, 2015: 30). Por ello, el gobierno relacionó el problema de "alimentar al pueblo" con la pobreza que reinaba en el campo. Haciendo uso de la demagogia característica de los regímenes políticos de la época, se propuso la justicia social para los campesinos que habían sobrellevado la carga de producir el alimento del país, es decir, se buscaría un reparto equitativo de los bienes sociales (Arteaga, 1985: 299). Además de la idea de justicia social para los campesinos, es cierto que el juego político marcaba una intervención en el campo para contrarrestar el descontento que había entre los campesinos. El discurso de renovar la alianza con el sector agrícola se orientó para mantener una estabilidad en el campo y para que el gobierno no perdiera la base de apoyo que representaban los campesinos.

Según Cassio Luiselli -quien fue director del SAM-, la crisis agrícola se vio recrudecida por el aumento en el consumo de proteína animal por las clases medias urbanas, además de las grandes extensiones de tierra que se habían destinado para la ganadería, el incremento de cultivos para la alimentación 
animal y la disminución de la producción de vegetales para consumo humano (Luiselli, 1982: 4-5). Con ello, el gobierno empezó a trabajar en una política pública que le permitiera maniobrar entre las demandas de los campesinos y el problema de impulsar la producción agrícola, para alcanzar la autosuficiencia alimentaria que tendría como resultado inmediato la baja en el gasto público por importaciones de granos básicos.

En 1979 fue el momento álgido de la crisis, de acuerdo a los datos que presenta Nora Lustig, cuando la superficie cosechada alcanzó el nivel más bajo de los últimos años (14,8 millones de hectáreas); también la composición de cultivos cambió al aumentarse el porcentaje de cultivos para consumo animal $(9,92 \%)$ y disminuyendo el de alimentos básicos $(51,80 \%)$, cuando en la década de 1960 estas cantidades fueron de 2,60\% y 72,60\% respectivamente (Lustig y Pérez, 1982: 264). A ello se agregó el decrecimiento de los precios de garantía del maíz y el fuerte aumento en importaciones en otros granos (trigo, frijol, sorgo), además de que la mayor parte de las divisas se gastaban en estas compras al exterior y el déficit productivo del sector agrícola no podía estabilizarse.

Es fundamental comprender este periodo de crisis agrícola y económica como una coyuntura heterogénea, que puso de nuevo en el escenario nacional al campo y de cierta forma evidenció que las promesas revolucionarias no habían sido cumplidas del todo, pues aquel reparto agrario tan efectivo en el gobierno de Lázaro Cárdenas había concluido hace mucho tiempo, dejando de lado a campesinos y ejidatarios y concentrándose en el desarrollo agroindustrial. Era necesaria una acción, la respuesta fue la creación del SAM.

\section{Sistema Alimentario Mexicano: producción, consumo y alimentación (1980-1982)}

Para 1980, año en el que se dio a conocer el SAM, ya había habido intentos de planificación de la actividad agropecuaria en México. Según Javier Arteaga, en febrero de 1975 se creó la Comisión Nacional Coordinadora del Sector Agropecuario (CONACOSA), encargada de administrar diversos proyectos de las dependencias gubernamentales relacionadas con las prácticas agrícolas (Arteaga, 1985: 299). Esta comisión tenía la labor de abastecer de productos básicos y de diseñar un Plan Nacional Agrícola que se preocuparía por lograr la autosuficiencia de alimentos básicos y establecer una estructura social orientada al desarrollo agropecuario.

A pesar de que la CONACOSA proponía algunas de las metas que el SAM retomaría, su acción y funcionamiento diferían de éste último, pues no contemplaba como problema esencial la crisis agrícola que se presentó tiempo después. Para fines de 1976, la situación agrícola del país era crítica, no solo 
por el peligro de la escasez de alimentos, sino por el enfrentamiento entre diversos sectores sociales por la tenencia y acceso a la tierra. Una vez en la presidencia, José López Portillo se enfrentó a una crisis política y a un déficit en la producción de granos básicos. Por ello, orientó su política social a la creación de empleos, aumento de salarios y a mejorar los niveles de vida de la población pauperizada. En 1977, para lograr su cometido, el gobierno puso en marcha el Plan Nacional Agropecuario, reminiscencia de la CONACOSA (EI Informador, 2-II-1977), el cual tenía el objetivo de incrementar la producción y alcanzar la autosuficiencia en maíz para el año 1979. Al mismo tiempo, con el afán de conciliar las demandas de las organizaciones agrícolas privadas y ejidales, se propuso una "Alianza para la producción", con la que se pretendía mediar entre los intereses y actividades de los productores, comerciantes e industriales agrícolas, con el fin de elevar la producción y ofrecer productos alimenticios a precios más bajos a la población en situación de pobreza ( $E$ I Informador, 31-V-1977).

En este ambiente de presiones de los sectores agrícolas y de la urgencia ante la crisis en el campo, el gobierno, desde la instancia del poder ejecutivo, resolvió presentar un proyecto cuya meta debía ser el autoabastecimiento de alimentos básicos. El 5 de agosto de 1980, el presidente José López Portillo se reunió con los gobernadores, los secretarios de Estado y con los principales dirigentes obreros y campesinos, para estructurar el plan de acción del SAM. En la reunión el presidente señaló:

"El Sistema Alimentario Mexicano es una estrategia inserta dentro del Plan Global de Desarrollo. ¿Qué queremos decir con estrategia? Usemos un símil del lenguaje bélico, en el que la estrategia es el arte de conducir los ejércitos al campo de batalla, para librarla con las tácticas. El Sistema Alimentario Mexicano quiere conducir las fuerzas de la nación, a dar la batalla por la alimentación suficiente de los mexicanos [...] El Sistema Alimentario Mexicano parte fundamentalmente de una fijación lógica de las metas: queremos una alimentación suficiente, aunque sea mínima, de todos los mexicanos" (López Portillo, 1980: 4-5).

De esta manera, el 18 de marzo de 1980, en el marco del cuadragésimo segundo aniversario de la expropiación petrolera, el presidente José López Portillo anunció tres cambios en su programa de gobierno. El primero fue el aplazamiento de la incorporación de México al Acuerdo General sobre Aranceles Aduaneros y Comercio (GATT, por sus siglas en inglés). El segundo se trató sobre el control de la producción diaria de petróleo y su venta a países extranjeros. El tercero fue la implementación del Sistema Alimentario Mexicano (SAM) como política que buscaría disminuir la dependencia alimentaria del país y sentar las bases de la autosuficiencia en alimentos básicos (Navarro, 1988: 115). 


\section{EI SAM: la apuesta política por la producción y la alimentación}

En el momento en que se dio a conocer el SAM tenía dos objetivos principales: un rápido aumento en la producción de alimentos básicos y apoyos múltiples al consumo de las mayorías empobrecidas de México (Spalding, 1985: 316-317). Sobre el incremento de la producción, el SAM se comprometía a proporcionar las condiciones materiales y financieras para lograr tal cometido, y en especial alcanzar la meta de autosuficiencia en la producción de maíz y frijol para el final del sexenio y en otros productos básicos (como el trigo) para 1985. En cuanto a la mejora de la distribución de alimentos al tercio de la población que se encontraba desnutrida, se planteó la utilización de un perfil actualizado de la situación nutricional del país y con un análisis en la estructura del consumo de alimentos (SAM, 1980a), ello con el fin de fijar y cuantificar metas alimenticias para sectores específicos de la población y regiones críticas definidas desde un punto de vista de nutrición y pobreza. Es fundamental observar cómo el discurso gubernamental empezaba a utilizar el discurso de autosuficiencia y seguridad alimentaria para justificar un programa de desarrollo social que tenía implicaciones más económicas que nutritivas. La importancia de estos compromisos es que el gobierno dio comienzo al diseño de una serie de proyectos que incidieran en todas las fases de la cadena alimentaria: producción, transporte, distribución y consumo (Spalding, 1985: 317).

Es difícil definir el SAM en tanto su naturaleza. Por un lado, algunos autores, como John Durston, sostienen que fue una orientación política más que un programa, debido a que no contó con una estructura institucional para ejecutar sus planes, y sí con un discurso que el gobierno imprimió a las acciones de las distintas dependencias en las que se basó (Durston, 1981: 6). Por otro lado, hay investigadores como Rose Spalding y Martha Navarro que respaldan la idea de una política fundamentada en un programa de reactivación agrícola y de medidas de seguridad alimentaria para la población (Spalding, 1985: 317; Navarro, 1988: 116). ${ }^{6}$ Ésta última idea es la que patenta también este trabajo, pues el SAM no puede verse como una simple coyuntura, sino como un planteamiento del gobierno federal de construir una política alimentaria basada en múltiples proyectos, que si bien estaban dirigidos en su mayoría a incrementar la producción agrícola, también tuvieron una preocupación por resolver la crisis alimentaria y nutricional del campo, para lo cual se elaboraron planes de difusión de ideas sobre una

6 El problema de definición del SAM es que muchas veces los autores toman programa, proyecto y política como sinónimos; sin embargo, el presente trabajo reúne estas tres denominaciones para hablar del SAM como una política que estuvo estructurada por diferentes programas de acción y proyectos de aplicación. 
alimentación saludable basada en la importancia de hacer incrementar la producción agrícola mexicana. ${ }^{7}$

Para dirigir eficientemente las acciones gubernamentales que allanarían el camino a la autosuficiencia alimentaria del país, se elaboraron metas de consumo que pudieran ser traducidas a metas de producción (SAM, 1980a: 9). Tal acción fue importante, porque hizo necesario el diseño de un perfil alimentario de México que estuviera actualizado y con un amplio alcance. Dicho perfil se construyó con ayuda del Instituto Nacional de la Nutrición (INN) durante la segunda mitad de 1979; se realizaron encuestas a más de 21 mil familias en 300 comunidades rurales y en las capitales y ciudades importantes de los estados. Con base en la información sobre consumo calórico y proteico que se sistematizó, se estableció que el mínimo normativo era de 2.750 calorías diarias, cantidad que era escasamente alcanzada por la mayoría de la población (SAM, 1980a: 9; Bourges y Casanueva, 2001: 186-188). De esta forma, se definió una "Población Objetivo" como una expresión dinámica que requería diferentes precisiones regionales. Dicha población abarcaba todas las personas que no podían cubrir los mínimos normativos nutricionales señalados por el INN de 2.750 calorías y 80 gramos de proteínas diarios; según los estudios para el año 1979, esta población era de 35 millones de mexicanos de un total poblacional de 70 millones para fines de esta década (SAM, 1980a: 9). Dada la amplitud poblacional fue indispensable delinear una "Población Objetivo Preferente" (POP), que ascendía a 13 millones de personas de áreas rurales y a 6 millones de zonas urbanas, las cuales se encontraban a un muy bajo nivel nutricional. A partir de esta información, el mismo INN se ocupó de señalar la distribución de la POP para identificar zonas críticas. Se encontró que éstas se hallaban en más de seiscientos municipios rurales y en las periferias urbanas, particularmente en la capital del país. Para aumentar las características de dichas zonas se analizaron otros elementos de bienestar, como salud, educación y vivienda.

A partir del perfil nutricional y de la identificación espacial de la PoP, se contrastó la encuesta ingreso-gasto de 1977 para encontrar el patrón de consumo prevaleciente en la población de menor ingreso. En esta canasta de consumo actual se identificaron tres tipos de alimentos: súper básicos (de mayor frecuencia) conformados por jitomate, frijol, cebolla, chile fresco,

7 Sobre las campañas de nutrición que el SAM diseñó a través de impresos y cápsulas radiofónicas, aún no hay trabajos que se encarguen de analizar dicha difusión. Queda pendiente resolver este vacío; solo el trabajo de Martha Navarro se acerca a estas campañas, sin embargo, la autora solo hace énfasis en el programa de educación nutricional que se llevó a cabo en algunas regiones del sureste mexicano (Navarro, 1988). En el texto de Jonathan Fox se analizan los consejos comunitarios, aunque el autor omite precisar que este programa inicialmente fue impulsado por el SAM, y sostiene la idea que fue la dupla COPLAMAR-CONASUPO la responsable de diseñar tales consejos (Fox, 1990). 
azúcar morena, maíz en grano, pasta para sopas, manteca de cerdo, arroz, café tostado, huevo, raíces feculentas, carne de res, aceite vegetal, pan dulce, pan blanco, leche bronca, plátano Tabasco y galletas; básicos (de frecuencia intermedia) constituidos por tortilla de maíz, carne de puerco, carne de aves, queso fresco, chocolate, té y otros tipos de leche; complementarios (de baja frecuencia) los cuales eran limón, leche pasteurizada, manzana, aguacate, harina de trigo, pescados y mariscos frescos o secos, naranja, harina de maíz, mantequilla, crema, lechuga y zanahoria (SAM, 1980a: 12). La configuración de esta canasta básica actual demuestra que para 1979 el grueso de la dieta mexicana se constituía por 38 productos que representaban el $86 \%$ del gasto del ingreso solo en alimentación, lo cual apuntaba a una pauperización de la población pues su consumo doméstico absorbía la mayoría de su ingreso, limitando otras necesidades como vestido, vivienda y recreación. Estos datos fueron importantes porque establecieron cuáles eran las necesidades cuantitativas de alimentos para la población objetivo y, de igual modo, cómo podían combinarse los productos para satisfacer los requerimientos nutricionales a bajo costo (SAM, 1980b).

Con base en las necesidades nutricionales de la población nacional, y teniendo en cuenta la diversidad regional en los hábitos de consumo y el poder adquisitivo, se estructuró la Canasta Básica Recomendable (CBR) a finales de 1979. La importancia de ésta fue que se convirtió en un instrumento de planificación económica que sirvió al Estado en su camino hacia la autosuficiencia alimentaria, ya que solventó la relación entre la satisfacción de las necesidades nutricionales de la población y la importancia del incremento de la producción agrícola. Igualmente, esta canasta fue importante en la política de subsidios que el gobierno implementó junto con el SAM. Para hacer llegar los alimentos de la CBR, el Estado tuvo que recurrir a la intervención de distintos canales de distribución de manera de hacer más eficiente la distribución. Con ese fin, utilizó la red de transportes y tiendas comunitarias que la CONASUPO tenía y empezó un proyecto de ampliación de carreteras y caminos secundarios para llegar a comunidades muy alejadas (SAM, 1980b). La aplicación del subsidio para que la gente pudiera adquirir a bajo costo la CBR fue a través del sistema DICONSA, el precio neto de la canasta era de 13 pesos, y con la subvención estatal se reducía un 30\%, quedando un precio de 9 pesos (SAM, 1980a; Spalding, 1985: 320). La difusión de la CBR se complementó con la campaña nacional de educación nutritiva, en la cual se hacía la promoción de la dieta tradicional mexicana, rica en fibras y productos naturales, en contra de los alimentos chatarra al ser identificados como productos industrializados ricos en harinas y azúcares (Navarro, 1988: 119). ${ }^{8}$ Una vez desarrollado el esfuerzo

8 Este es el único trabajo que se ha hecho sobre la campaña nacional de educación nutritiva; en él la autora relata cómo la puesta en marcha del SAM provocó que el Estado volviera a 
por transformar las deficiencias alimentarias en metas específicas de consumo, se planteó la necesidad de lograr la autosuficiencia a través de la producción de los alimentos básicos que conformaban la dieta ideal que se propugnó en la CBR; este hecho llevó a plantear un proyecto de ajuste e impulso al campo.

El plan estratégico del SAM sostiene claramente que su mayor prioridad era el logro de una adecuada y autosostenida producción y consumo de alimentos populares, con un propósito redistributivo (SAM, 1980c). Por ello, no puede asegurarse que la meta final del Estado era proporcionar justicia social a los campesinos, sino que iban implícitos intereses económicos y políticos. Esta idea parte de las mismas aseveraciones del planteamiento original del SAM. En él se esbozó la reorientación del consumo de las mayorías hacia la satisfacción de sus mínimas necesidades de bienestar, la cual no debía basarse solamente por la vía de producir y distribuir masivamente alimentos, sino que el fundamento debía ser el rescate de la agricultura nacional, la estimulación de la actividad piscícola y la ampliación del mercado interno. Para lograr tales objetivos generales se planteó la creación de empleos rurales, lo que llevaría a la mejor distribución del ingreso. Estas medidas expresan la conexión e influencia que tuvieron las recomendaciones hechas por la FAO, para llegar a la autosuficiencia en alimentos básicos.

Asimismo, los lineamientos generales de esta política apuntaban a la impostergable necesidad de soberanía alimentaria en cereales y oleaginosas, debido al grave contexto internacional de crisis agrícola, donde el "poder alimentario" sería utilizado como una ventaja frente a la favorable posición energética en la que se encontraba México. Es interesante constatar que el mismo plan general argumentaba que la búsqueda de la autosuficiencia alimentaria no estaba en contra de la economía exportadora del país, sino que era necesario hacer valer el sobrado potencial para ser autosuficiente en granos sin penalizar la producción de otros bienes (SAM, 1980c).

\section{Planteamiento, medidas y efectos programáticos del SAM}

Pare entender el funcionamiento de esta política alimentaria y comprender su influencia en la historia de la alimentación mexicana, resulta importante describir cuál fue su planteamiento estratégico, ya que a partir de éste coordinó sus operaciones. EI SAM partió de dos premisas fundamentales. En primer lugar, las divisas generadas por la exportación de petróleo permitirían la ampliación de la base productiva y del mercado interno. En segundo lugar, el Estado compartió los riesgos que conllevaban la inversión de la producción agropecuaria (Lustig y Pérez, 1982: 266). Por ello, es posible asegurar que las

poner atención en la nutrición como ciencia que contribuía al bienestar social. 
razones que el gobierno tuvo para considerar el problema alimentario como prioritario fueron más de carácter económico y de soberanía que de justicia social, es decir, no buscaban incentivar un nuevo reparto equitativo que ya se había hecho en los primeros gobiernos revolucionarios. Tal aseveración resulta consecuente con la declaración que hizo el gobierno en torno a que la autosuficiencia no significaba una autarquía, sino una ampliación de las condiciones productivas nacionales.

De ahí que la base esencial de la estrategia de la autosuficiencia alimentaria estaba en incrementar la actividad productiva en los distritos de temporal a través de la utilización de insumos que les permitirían aprovechar todo el potencial productivo de los recursos con los que contaban, por ejemplo, tierra, instrumentos de labranza y la fuerza de trabajo (SAM, 1980d). Hay que entender que la implementación de esta política no se trataba solo de producir masivamente alimentos, sino de que fueran producidos principalmente por campesinos de temporal, porque se aseguraba que eran los de mayor potencial de respuesta productiva y, con ello, se podría ofrecer empleo e ingreso a miles de trabajadores agrícolas, logrando que su reactivación productiva ayudara en la resolución de los problemas de subalimentación (SAM, 1979). Esta base estratégica les permitiría a los campesinos quedarse con el excedente generado por el aumento de la productividad; por lo tanto, el Estado debía poner en marcha políticas específicas para poder vigilar el proceso productivo en todas sus etapas. Cabe señalar que esta estrategia del SAM no representaba la inducción a una rápida modernización, al introducir maquinaria o implementos con las que las unidades de producción campesina no estuvieran familiarizadas ni preparadas para adquirir o controlar, sino que se trató de incrementar la productividad utilizando fertilizantes, semillas mejoradas y plaguicidas.

Tales acciones guardan una estrecha relación con las condiciones que la Revolución Verde incentivó a partir de la década de 1960 en gran parte del mundo, con el fin de incrementar la productividad agrícola y de alimentos. En México, este proceso influyó en la reconfiguración de una parte del agro mexicano, pues el Estado aplicó dicho paquete de acciones a favor del incremento de la productividad y del rendimiento agrícola (Pichardo, 2006: 41), y en donde se evidenció fue en el caso del SAM, pues su planteamiento general y sus metas de consumo dependían del éxito en el incremento de la producción de alimentos básicos en el campo.

Con estos argumentos, es posible empezar a describir cuáles fueron las medidas operativas del SAM. Aunque muchas ya se han apuntado parcialmente, es necesario señalarlas para comprender cómo se pretendió apoyar la producción de alimentos básicos para alcanzar la autosuficiencia alimentaria durante el periodo de primavera-verano de 1980, y resolver el problema de subalimentación en el campo. Las medidas más importantes, desde el punto 
de vista económico, fueron los precios de garantía y los subsidios a los insumos; dicha disposición se proponía hacer más competitiva la producción de alimentos básicos para asegurarle al productor un ingreso y protegerlo de la inflación (Luiselli, 1982: 20). Así, hubo subsidios para que los campesinos pudieran comprar diversos insumos para intensificar la producción. Por eso fue necesaria la participación de otras empresas estatales como Fertilizantes Mexicanos (FERTIMEX) que se encargaría de importar fertilizantes y plaguicidas, principalmente de Estados Unidos, para ponerlos al alcance de los productores a un precio reducido y fijo; la Productora Nacional de Semillas (PRONASE), la cual tendría la tarea de producir semillas mejoradas y hacérselas llegar a los campesinos (Excélsior, 11-VII-1981); y quizás el caso más icónico de subsidios fue el que desempeñó la Compañía Nacional de Subsistencias Populares (CONASUPO): esta dependencia revivió el Programa de Apoyo a la Comercialización Rural (PACE), con el cual promovió compras de maíz a ejidatarios, además de haber ampliado sus actividades de promoción de la Canasta Básica Recomendable y de una dieta balanceada estructurada a partir de los alimentos que subsidiaba (Spalding, 1985: 330; Navarro, 1988: 133-135).

Otra de las medidas operativas del SAM fue el crédito para la producción a través del Banco Nacional de Crédito Rural (BANRURAL); estos recursos financieros y crediticios se estimarían en función de la producción de alimentos básicos, tomando en cuenta el nivel técnico de cada zona, estrato de productores y las necesidades técnicas del terreno (SAM, 1980d; Lustig y Pérez, 1982: 267). En relación con esta medida, también se aplicó el seguro agrícola, bajo la dirección de la Aseguradora Nacional Agrícola y Ganadera; ésta cubriría la totalidad de las cuotas de crédito, desde la preparación de la tierra hasta los transportes para la cosecha, protegiendo al agricultor de las pérdidas parciales o totales que pudieran provocar factores externos como el clima (Excélsior, 31-V-1981; 4-VII-1981).

A pesar de que ya se esbozó anteriormente, es importante recalcar la importancia que tuvo el programa de riesgo compartido, pues constituyó uno de los principales instrumentos de apoyo del SAM. Esta medida partió del hecho de que los campesinos de temporal trataban de minimizar las probabilidades de pérdida de sus cosechas y trataban de maximizar sus ganancias para que su subsistencia alimentaria no fuera comprometida. La incorporación de nuevas tecnologías que proponía el SAM para incrementar la producción conllevaba muchos riesgos, por lo que esta medida consistió en el establecimiento de una alianza entre el Estado y los campesinos de temporal, para el aumento de la productividad en maíz y frijol (SAM, 1980d).

La ampliación de la frontera agrícola también fue otra de las medidas; buscaba la reincorporación de tierras, en especial aquellas con potencial pro- 
ductivo que estuvieran en manos del sector ganadero y aquellas con capacidad agrícola a través del riego; además, se buscó utilizar terrenos por medio del desmonte. Asimismo, fue importante ampliar la actividad piscícola debido a que el pescado contaba con un alto valor nutritivo y su precio por gramo de proteína era más bajo entre los alimentos de origen animal. El plan de pesca y el fomento a la acuicultura en zonas rurales empobrecidas fue importante para la obtención de este producto, lo que contribuyó a que varias especies de pescado de agua dulce se incorporaran a la Canasta Básica Recomendable, arguyendo su contenido nutricional (Luiselli, 1982: 22).

\section{Campañas por la alimentación}

Estas medidas operativas se implementaron en conjunto con varios proyectos de desarrollo agrícola que se diseñaron desde el SAM. A pesar de que fueron más de veinte proyectos los que se presentaron, debido al poco tiempo de operación del SAM no todos se llevaron a cabo. Algunos ya fueron mencionados, por ejemplo, la creación del perfil nutricional y la estructuración de la CBR, no obstante, queda por explicar la promoción y educación para la producción y el consumo, en concomitancia con la campaña nacional de educación nutritiva. Esta última estuvo integrada por cuatro subcampañas que surgieron del argumento de que no era suficiente subsidiar y recomendar los alimentos de la CBR, sino que también era necesario hacer una difusión constante de estos esfuerzos por corregir hábitos alimentarios y resolver el problema de la desnutrición en el país. Según Cassio Luiselli, se creyó que una campaña en los medios de comunicación masiva era lo ideal, pues tales mecanismos -prensa, radio y televisión- tenían la capacidad de incidir en los patrones de gasto y consumo de la población (Luiselli, 1982: 14). Así que se promovería en éstos la dieta tradicional mexicana en contra de la comida chatarra. El gobierno se encontró ante una disyuntiva política y económica. Por un lado, podía combatir la publicidad y expansión de los alimentos industrializados y dañinos al regular el contenido de los anuncios de dichos productos; por otro, podía crear sus propios anuncios con mensajes de salud y nutrición en la alimentación de niños, jóvenes y mujeres embarazadas y en lactancia. Está claro que prefirió esta última alternativa.

La primera campaña dio arranque en 1980, estuvo dedicada a la importancia de la leche materna y se tituló Lo que la madre debe saber sobre su alimentación y la de su hijo. En ella se presentaban recomendaciones sobre las ventajas de la lactancia de pecho, los riesgos de las leches industrializadas, así como dietas recomendadas para niños recién nacidos y de uno a cinco años. Se promovieron mensajes como "la buena alimentación empieza antes de nacer" o "de la adecuada alimentación de la madre dependen: su salud, el desarrollo intrauterino de su hijo y la pos- 
terior producción láctea" (SAM, 1980e: 5). La segunda campaña también fue puesta en marcha en 1980 y se basó en la distribución de recetarios, carteles, calendarios y cuadernillos para hacer explícitos y públicos los valores nutritivos de la dieta mexicana, así como la importancia del SAM en el problema alimentario del país. ${ }^{9}$ Una tercera campaña se realizó en 1981 y se basó en la difusión de mensajes radiofónicos (algunos en lenguas indígenas) y segmentos televisivos que trataban sobre la importancia de comer bien (Luiselli, 1982: 15-16). Además, hubo una cuarta campaña en ese mismo año dedicada a editar un libro familiar sobre el buen comer, también se incluyó en los libros de texto gratuito información sobre los efectos nocivos de la comida chatarra y la explicación de la importancia de una buena salud basada en una alimentación balanceada en calorías y proteínas. A esta difusión se le agregaron las brigadas del Desarrollo Integral de la Familia (DIF) en zonas rurales, compuestas por nutriólogos, para difundir hábitos alimentarios sanos y nutritivos (Luiselli, 1982: 1516). Estos esfuerzos fueron la acción social que el Estado ejerció como disposición para apoyar y fomentar el modelo de dieta que había diseñado como respuesta ante la crisis agrícola y la subalimentación que sufría gran parte de la población mexicana.

Esta serie de medidas operativas y proyectos tenían el objetivo de incentivar a los agricultores mediante la disminución de costos de las tecnologías y que fueran empleadas de manera más rápida, lo que ayudaría a modernizar el campo y, con ello, la producción de alimentos básicos; del mismo modo, se pretendía inculcar a la población valores nutritivos a partir de la difusión en radio, televisión y prensa de hábitos alimentarios "sanos y saludables" que estuvieran acordes a los requerimientos productivos que el país necesitaba cubrir. Estas acciones harían que el Estado ganara dos frentes: superaría la crisis agrícola y el rezago en el agro mexicano. Sin embargo, ¿el SAM logró la victoria en estos dos frentes? ¿Qué problemas tuvo que enfrentar en su intento por llegar a la autosuficiencia alimentaria y la reactivación del campo? Para contestar estas cuestiones es necesario comprender que los impactos del SAM, aunque fueron mesurados, respondieron a una situación política específica, en la que la conciliación de grupos de intereses económicos y aquellos que apostaron por las reformas estructurales para solucionar la crisis alimentaria del país llevó a una lucha interna por la primacía en la toma de decisiones.

9 Un ejemplo de esto fueron los cuadernillos para el campesino que las autoridades del SAM otorgaron gratuitamente, en los cuales venían lecciones sobre los cultivos con semillas mejoradas y por qué era importante producir para alimentarse mejor. 


\section{Lucha por la alimentación: el SAM y sus obstáculos}

Muchos de los problemas políticos y administrativos a los que se enfrentó el SAM, así como su misma liquidación, provinieron de la estructura gubernamental a la que pertenecía. En el anuncio de su creación, el SAM contó con el apoyo incondicional del presidente José López Portillo, mientras que las críticas al interior del gobierno fueron silenciadas (Redclift, 1981: 7). Sin embargo, la respuesta de los empresarios de la Confederación de Cámaras Industriales (CONCAMIN) no se hizo esperar, pues veían en esta política una amenaza a su estatus de "consentidos del régimen"; les preocupaba que las concesiones e inversiones del Estado se trasladaran en su mayoría a las actividades agropecuarias (Redclift, 1981: 7). En cambio la Confederación Nacional Campesina (CNC) vio con buenos ojos esta estrategia gubernamental y ofreció apoyo al Estado para poder llevarla a buen puerto. De esta manera, el SAM, como política estatal para enfrentar la crisis productiva, surge como propuesta del ejecutivo federal para conciliar producción y alimentación.

La concepción neutral y fuera de la estructura burocrática que tuvo el SAM fue una de sus mejores características, porque ello significó una autonomía de las dependencias gubernamentales que ya trabajaban en asuntos del campo, y se limitó solo a usarlas en la ejecución de sus programas y proyectos. La participación que propició el SAM de diversas organizaciones políticas e instancias gubernamentales para solventar el proyecto de autosuficiencia alimentaria unió en un mismo proyecto a la Secretaría de Agricultura y Recursos Hidráulico (SARH), la Secretaría de la Reforma Agraria (SRA) y la Secretaría de Programación y Presupuesto (SPP), quienes se encargaron de elaborar el Plan Nacional de Desarrollo Agropecuario e impulsar la Ley de Fomento Agropecuario en 1980, ambos destinados a normar las metas de producción de alimentos básicos para los últimos años del sexenio de López Portillo (Arteaga, 1985: 304-305). Además, participaron otras organizaciones y empresas estatales, que ayudaron al proyecto al brindar recursos financieros, propaganda y subsidios; como ya se indicó, algunas de ellas fueron la CONASUPO, BANRURAL y la CNC.

La Ley de Fomento Agropecuario (LFA) encerraba una contradicción con las propuestas del SAM, ya que esta ley buscaba incorporar al cultivo todas las tierras susceptibles de explotación y fomentar la organización campesina para la producción, por medio de comités directivos en cada distrito de temporal (Arteaga, 1985: 307). La contradicción se hallaba en que esta norma permitía la asociación entre ejidatarios y pequeños propietarios con el fin de incrementar la producción en el menor tiempo posible. Este cambio en la forma de asociación, si bien no afectaba las formas de tenencia de la tierra, abría una brecha para que muchos propietarios pudieran arrendar tierras a ejidatarios y expandir sus terrenos de cultivo. Esto provocaría que la 
agricultura de temporal también se orientara al comercio externo, desfavoreciendo la producción interna y la autosuficiencia alimentaria del país. Por eso, es posible afirmar que la unión entre el SAM y la LFA marcó el triunfo del sector pequeño propietario, el que se beneficiaba más que los campesinos ejidatarios, comuneros y jornaleros. Si bien el SAM trató de cumplir con dos acciones específicas para incidir en el incremento de la producción, que fueron capitalizar el campo (mediante la tecnificación y el crédito) y aliviar las tensiones sociales y políticas que generó el agotamiento de la política de reparto agrario, no logró suprimir el descontento social, sino que repitió, como otras políticas gubernamentales, el papel de mediador entre grupos de interés y los sectores populares, ya que el Estado cambió su orientación de repartir tierras hacia la búsqueda de la productividad bajo el lema de la autosuficiencia alimentaria.

Otro elemento que abonó al deterioro del SAM fue la competencia burocrática. A partir de 1981, los directivos del programa tuvieron desencuentros con los encargados de la SPP, debido a la forma en que se pretendían administrar los subsidios y las compras a través de la CONASUPO; esto solo reflejó el poco poder de acción que los encargados del SAM tenían. A pesar de ser independientes y contar con el apoyo del presidente, no pudieron hacer frente a los obstáculos que otras instancias les ponían (Excélsior, 29-IV-1981; Spalding, 1985: 331). También aparecieron las quejas de la industria de procesamiento de alimentos, pues esta rama había experimentado un creciente proceso de transnacionalización en productos como leche condensada, té, refrescos, colorantes, café instantáneo y frituras, por lo que las campañas en contra de este tipo de artículos que alentó el SAM no fueron de su agrado, alegando que solo se dañaría la economía del país, aunque estaba claro que la dieta promovida en dichas campañas solo hacía quedar a los productos procesados frente a la población como artículos comerciales sin valor nutritivo e incluso nocivos para la salud, información que era validada por estudios y análisis del Instituto Nacional de Nutrición.

El fin del SAM llegó junto con la crisis fiscal de 1982, debido a que el Estado perdió el poder para seguir financiando diversos programas de bienestar social, y los organismos internacionales que intervinieron para aminorar el golpe económico en México tuvieron el poder de decidir los nuevos lineamientos de la política económica que se implementaría para la recuperación. Uno de estos organismos fue el Fondo Monetario Internacional (FMI), el cual concedió al país un préstamo bajo la condición de adoptar un paquete de políticas económicas ortodoxas; estas incluían supresión de subsidios gubernamentales, flotación de precios y devaluación de la moneda. Por lo tanto, el SAM era totalmente incompatible con la nueva realidad económica, debido a que sus proyectos implicaban una serie de subsidios y créditos para los productores, además de los precios de garantía. Esta situación se agudizó en el 
momento en que el país empezó de nuevo a importar granos básicos -maíz y frijol- desde Estados Unidos. El renovado pacto comercial tuvo ventajas para ambos países. Para el gobierno estadounidense significó poder hacer frente a la crisis de sobreproducción de su sector agrícola, pues con las medidas de expansión de fronteras agrícolas y la utilización de las semillas mejoradas, fomentadas por la Revolución Verde, necesitaba un mercado el que enviar sus excedentes. Para México significó poder desarrollar un nuevo sistema de distribución, esta vez a manos de la CONASUPO, que lograra hacer llegar los alimentos importados al mercado interno.

Otra de las posibles explicaciones del fallo del SAM es que el gobierno lanzó esta política sin tener una vinculación sólida con el campesinado y sin constituir un organismo representativo para los beneficiarios. De igual forma, no vinculó los movimientos campesinos que reivindicaban la reforma agraria, ya que, como se indicó, el SAM estimuló la producción y no el reparto de tierras; esta situación hizo que la política careciera de las bases campesinas, por lo cual no contó con defensores ante los detractores que incitaron su pronta desaparición.

En cuanto a uno de los probables impactos que tuvo el SAM en el campo mexicano, uno de ellos fue el estímulo de nuevas formas de participación popular para obtener acceso a la alimentación. Esta participación se dio a través de la movilización de los campesinos de temporal, que empezaron a enarbolar demandas sociales, particularmente sobre la producción de alimentos básicos y la importancia que tenían para la subsistencia de miles de familias campesinas. La situación propició que se empezara a crear consejos comunitarios encargados de vigilar la distribución de los insumos y de los créditos, así como de los alimentos subsidiados. Jonathan Fox apunta que tales consejos se dan con el proyecto entre COPLAMAR-CONASUPO y define al SAM como una "revolución desde arriba", auspiciada por la élite política del momento (Fox, 1990: 41). Sin embargo, no precisa que fue en el marco de los proyectos de difusión y modernización del campo del SAM cuando se inicia un proceso de politización en las comunidades rurales (Excélsior, 26-IV-1981).

El decaimiento del SAM llegó el 30 de noviembre de 1982, tan solo con dos años de operaciones. Sus programas empezaron a perder eficacia, al igual que la red de subsidios que había creado. Esta situación se debió presuntamente a la nueva política económica que se instauró para hacer frente a la crisis económica desencadenada por la caída de los precios del petróleo y que sumió al país en una depresión financiera muy fuerte. A pesar que se aseguró que su estrategia central se mantendría y que algunos de sus programas serían adaptados y reubicados en distintas dependencias, esto no sucedió. Hubo un nuevo proyecto para seguir adelante con el apoyo a la producción, distribución y consumo de alimentos básicos, éste fue el 
Programa Nacional de Alimentación (PRONAL), lanzado por el gobierno de Miguel de la Madrid en 1983. Sin embargo, fue un planteamiento que no aglutinó los programas de desarrollo agrícola que contempló el SAM, pues el presupuesto con el que contó fue mucho menor y su capacidad de acción estuvo limitada por la nueva orientación económica del gobierno, en la cual la reducción del gasto social fue marcada, dejando de lado programas de beneficios sociales.

De esta manera, la crisis económica coincidió con la salida del presidente López Portillo, lo que acarreó duras críticas a su administración y por las circunstancias tan graves en las que dejaba al país. La prensa empezó a atacar las políticas sociales aplicadas en el sexenio que terminaba, identificando al SAM como el mayor fracaso para combatir la pobreza y la crisis agrícola (Proceso, 17-I-1983; Spalding, 1985: 339-340). Después de dos años de buscar, a través de una política innovadora, la autosuficiencia alimentaria y la eficiencia productiva del campo, México volvía a importar una parte importante de los alimentos básicos para alimentar a su población.

\section{Consideraciones finales}

La importancia que subyace en el análisis del SAM es la comprensión de un periodo turbulento en las políticas públicas de México. Además, es importante abordar el debate que existió sobre este programa y sus efectos en la lucha por alcanzar la autosuficiencia de alimentos básicos en un momento en el que la escasez de insumos era la preocupación principal en el mundo. Las ideas que se han planteado en este trabajo están dirigidas a renovar la idea sobre las causas que llevaron al diseño de esta política, así como para analizar los objetivos que enarboló para solventar el déficit alimentario.

De igual manera, se considera que el SAM es una expresión de la forma en que el Estado enfrentó, durante las décadas de 1970 y 1980, el problema alimentario de México, el cual no solo consistía en los problemas del campo (baja producción, poco acceso a la tierra), sino en el ascenso de los problemas nutricionales en la población rural y de periferias urbanas, así como en el incremento de los precios de productos básicos. Por esta razón, se concluye que el análisis del SAM, junto con sus antecedentes, objetivos, programas y recursos gubernamentales e institucionales, permite conocer una parte de la historia contemporánea de la alimentación en México. Asimismo, el análisis de dicho programa resulta fundamental para comprender los procesos sociales que contribuyeron en la elaboración y puesta en práctica de un proyecto de fomento agrícola, mismo que no surge solo de la coyuntura de crisis alimentaria, sino por intereses económicos y políticos relacionados con el sistema productivo mexicano. 


\section{Referencias bibliográficas}

\section{Fuentes primarias}

a) Archivos

Biblioteca Central de la Universidad Autónoma Chapingo.

Biblioteca Daniel Cosío Villegas - El Colegio de México.

Hemeroteca Nacional, México.

b) Publicaciones periódicas

El Universal, Ciudad de México, 1935.

El Informador, Guadalajara, 1977.

Excélsior, Ciudad de México, 1981.

Proceso, Ciudad de México, 1983.

\section{c) Publicaciones oficiales}

Sistema Alimentario Mexicano (1979). Notas analíticas y Lineamientos Metodológicos del Sistema Alimentario Mexicano.

Sistema Alimentario Mexicano (1980). Sistema Alimentario Mexicano: primer planteamiento de metas de consumo y estrategia de producción de alimentos básicos (1980-1982).

Sistema Alimentario Mexicano (1980). Medidas Operativas, Agropecuarias y Pesqueras, Estrategia de Comercialización, Transformación, Distribución y Consumo de los Productos de la Canasta Básica Recomendable.

Sistema Alimentario Mexicano (1980). Estrategia de Comunicación Social. México.

Sistema Alimentario Mexicano (1980). La estrategia temporalera del Sistema Alimentario Mexicano y su operación.

Sistema Alimentario Mexicano (1980). Lo que la madre debe saber sobre su alimentación y la de su hijo. Dirección de Publicaciones de la Comisión General de Comunicación Social. 


\section{Fuentes secundarias}

\section{a) Artículos y capítulos de libros}

Arteaga, J. (1985). "El Sistema Alimentario Mexicano (SAM): una perspectiva política", en Estudios Sociológicos, Vol. 3, No. 8, Los alimentos en la sociedad: aportes al estudio interdisciplinario de la alimentación, (May. - Ago.), COLMEX, pp. 297-313.

Bourges, H. y E. Casanueva (2001). "Reseña histórica sobre la Nutriología en México", en Bourges, H., J. Bengoa y A. O'Donell (coords.), Historias de la Nutrición en América Latina. México: Sociedad Latinoamericana de Nutrición (SLAN), Fundación Cavendes-INCMNSZ-CESNI, pp. 176-216.

Durston, J. (1981). "El Sistema Alimentario Mexicano (SAM): ¿un nuevo estilo de desarrollo social rural?", en Documento de discusión para la División de Desarrollo Social de la Comisión Económica para América Latina (CEPAL), pp. 1-32.

Fox, J. (1990). "La participación popular y el acceso a la alimentación: los consejos comunitarios de abasto en México, 1979-1986", en Investigación Económica, Vol. 49, núm. 191 (enero-marzo). México: UNAM, pp. 33-66.

López, J. (1980). Sistema Alimentario Mexicano, Cuadernos de filosofía política 36. México: Secretaría de Programación y Presupuesto.

Luiselli, C. (1982). "The Mexican Food System: elements of a program of accelerated production of basic foodstuff in Mexico", en Research report series, 22. San Diego: Center for U.S.-Mexican Studies, University of California, pp. 1-24.

Lustig, N., y R. Pérez (1982) "Sistema alimentario mexicano: antecedentes, características, estrategias y efectos" en Problemas de Desarrollo. Revista Latinoamericana de Economía, Vol. 13, núm. 51/52, pp. 247-286.

O'Broin, S. (2015). "Historia de la FAO en siete décadas", en Pedro Javaloyes (coord.), 70 años de la FAO (1945-2015), FAO, pp. 12-79.

Pichardo, B. (2006). "La Revolución Verde en México", en Agrária, Sao Paulo, $N^{\circ} 4$, pp. 40-68.

Redclift, M. (1981). "Development policymaking in Mexico: the SAM", en Working papers, Institute of Latin American Studies, University of London, pp. 1-12.

Spalding, R. (1985). "El Sistema Alimentario Mexicano (SAM): ascenso y decadencia", en Estudios Sociológicos, Vol. 3, núm. 8, Los alimentos en la sociedad: aportes al estudio interdisciplinario de la alimentación, (May. - Ago.), COLMEX, pp. 315-349.

Yúnez, A. (2010). "Las transformaciones del campo y el papel de las políticas públicas: 1929-2008," en Kuntz, S. (coord.), Historia económica general de México. De la Colonia a nuestros días. México: El Colegio de México, pp. 729-755. 


\section{Libros}

Cárdenas, E. (2015). El largo curso de la economía mexicana. De 1780 a nuestros días. México: El Colegio de México-Fondo de Cultura Económica.

Hewitt, C. (1988). La modernización de la agricultura mexicana, 1940-1970. México: Siglo XXI Editores.

Luiselli, C. (2017). Agricultura y alimentación en México. Evolución, desempeño y perspectivas. México: UNAM-Siglo XXI Editores.

Navarro, M. (1988). Development policy and nutrition: A case study of the nutrition education campaign of the Sistema Alimentario Mexicano (SAM). Stanford: Stanford University.

\section{Cómo citar este artículo:}

Pedroza Ortega, L. O. (2018) "El Sistema Alimentario Mexicano: su acción en el campo y en la alimentación, 1980-1982", en Revista de Historia y Geografía, №39, pp. 21-48, Escuela de Educación en Historia y Geografía, Universidad Católica Silva Henríquez (UCSH), Santiago de Chile.

[http://ediciones.ucsh.cl/ojs/index.php/RHyG/article/view/1691/1553] 